\title{
Personal information environment awareness in the Czech Republic
}

\author{
Pavel Valášek ${ }^{1, *}$ \\ ${ }^{1}$ Tomas Bata University in Zlin, nam. T.G. Masaryka 5555, 76001 Zlín, Czech Republic
}

\begin{abstract}
This article discusses the condition of information and communication technology usage in relation to problematics of the personal information environment in the Czech Republic. As to start any broader study of this topic in the ara of the Czech Republic, it is necessary to assess a current perception of the personal information environment and its elements. As existing studies are aimed mainly at a technical point of view, the presented study is focused on user participation a perception of individual technology elements. By a mean of the survey, a data were collected and analyzed using cluster analysis. Established groups of users are presented. The results of this study may create the suitable basis for a further research of discussed phenomena in the Czech Republic as well as an element of comparison for similar studies in other countries.
\end{abstract}

\section{Introduction}

Along with the constant development of information and communication technology (ICT), its general public accessibility rises. Exploration of this trend in the Czech Republic reveals a gradual increase in a number of households equipped with some form of a personal computer (desktop, notebook).

Surveys conducted between years 1991 and 2015 show an increase in the number of Czech households equipped with a personal computer from 100 thousand households (4\% population of Czech Republic) to almost 3.2 million of households (73\% population of Czech Republic) in the 2nd quarter of 2015. [1, 2, 3, 4]

This development can correspond with an average number of households with the internet access among the states of European Union, where the biggest portion of households with a high-speed internet connection was found in Netherlands, Luxembourg, Denmark, and Sweden. The lowest number was found in Bulgaria: only $54 \%$ of households have a personal computer and use it for the possibility of internet connection. However, the Czech Republic along with Romania, Bulgaria, Hungary, Slovakia, Poland, and Greece is one of the countries with the highest growth in the number of personal computers with internet connection between the observed years 2007 and 2015. [1, 2, 3, 4]

These technologies facilitate not only an easier access to information, its inspection, processing and archiving, but also present new activities, services, and other possibilities (for example social networks and data storage). Depending on a point of view, it can be asserted that the value of information has increased as well as its effect on the quality of life and tendencies of social, economic and political processes. Currently, digital information is considered to be the main medium due to its support of information extraction and preservation. $[5,6,7]$

The fastest and most effective information processes for the benefit of an organization or an individual are the current global focus of this field. Information technology and informatics alone cannot complete this task and therefore other scientific disciplines have to be involved, for example, findings from areas of psychology, humancomputer interaction (HCI) or librarianship. An individual, consciously or subconsciously, creates a set of elements with a purpose of extraction and processing of information. This structure can be identified as a personal information environment information environment (PIE). It contains all information sources, tools and methods involved in information processes that the individual encounters and regularly uses. Consistently with the current trend of the ICT development, the influence of new technology elements can be observed.

So far conceptions of the informational environment did not consider a user or a human element in general, as one of the key aspects of this problematics. The main emphasis of the existing research was the technical and technological development in the field. However, it should be always remembered that in the end, it is a user, a human, who operates the whole system and interprets its results. Therefore the system is only as efficient, 
functional or "good" in general as its user allows it to be. Improvements in the availability of ICT and inclusion of ICT into everyday lives helped reveal some user related phenomena. Unfortunately, mostly only marginal or highly specific studies were conducted to this day.

Considering these reasons, the aim of this study was to explore the current situation in the area of PIE and to determine what elements of ICT are used the most and how they are used. Finally, the distribution of non-ICT information sources and processes was also explored. Data collection for purposes of the study was performed in a form of a semi-structured interview. This option eliminated the possibility of inaccuracies caused by miscommunication. Collected data were analyzed and user groups were established, creating new ways of user categorization. By outlining these basic guidelines, a great progress can be achieved. Users can be sorted by their stance toward ICT, by their proficiency and preferences or other aspects. It enables us to see new ways of designing complex systems of both ICT and non-ICT elements with specific groups of users in mind. This new strategy can be used in any field that utilizes elements of ICT, but most notably in various security applications.

\section{Methods}

As a part of a broader study, a survey was conducted with an aim on personal information environment. The main topic of the survey was divided into three subsections: covering fields of an information environment and its relation to an individual user, the perception of information fragmentation problems and strategies of information fragmentation coping.

The first section, exploring a structure of the current information environment of individuals, is focused on an enumeration of information and communication technology elements of an individual. Its questions address actively used forms of computers, quantity, and character of data storages as well as applications used for a process of creating information. The objective of the section called "perception of information fragmentation " is to reveal a level of general awareness considering a topic of information fragmentation, more specifically if users encounter and realize problems of information fragmentation and if they feel any need to react to related issues. It is closely related to the next section "strategies of information fragmentation coping". The topic is specified by an effort to establish specific methods and technologies used to eliminate or at least lighten the issues related to the information fragmentation.

Because of the sophistication of the conducted survey, a suitable number and composition of respondents had to be chosen. A group of 52 second year "informatics in administration" students were selected. These particular students' specialization and stage of education were selected because they ensured a certain level of previous knowledge of information and communication technologies. Students were selected in this group by means of convenient sampling. The questionnaire itself was personally administered, consisting of ten open-ended questions from this field. The collected data were evaluated using cluster analysis. Final results in a form of user groups were established and a provisional name for each group was selected, according to a major characteristic of such group.

The main difference between existing studies is the focus of questions. The aim of this study is at the user ad its perception of mentioned elements and their usage opposing to an examination of these elements in other studies.

\section{Results}

Survey results evaluation reveals that up to $52 \%$ of users are aware of the risks associated with the information fragmentation (IF) phenomenon and try to prevent them. Other users either do not perceive these problems or do not use any form of strategy when dealing with this kind of problems. [8]

Because ICT in this context is seen as one of the motivating elements of ongoing research one of the questions was aimed at ICT elements they use. It was expected that the personal computer would be the most common ICT element among users. But the collected data shows that the most used ICT element is a notebook (or laptop). More than half of all respondents own a notebook (64\%) but only about one-fifth of asked users (19\%) regularly use a personal computer. When asked about why they prefer a notebook, their responses concerned mainly mobility in comparison to a personal computer and better functionality in comparison to a smartphone. Respective percentages of respondents using respective devices can be seen in presented figure (Fig. 1). Discussed ICT elements are marked as following:

$$
\begin{array}{ll}
\text { - } & \text { PC - personal computer } \\
\text { - } & \text { NB - notebook (laptop) } \\
\text { - } & \text { SP - smartphone } \\
\text { - } & \text { O - other }
\end{array}
$$

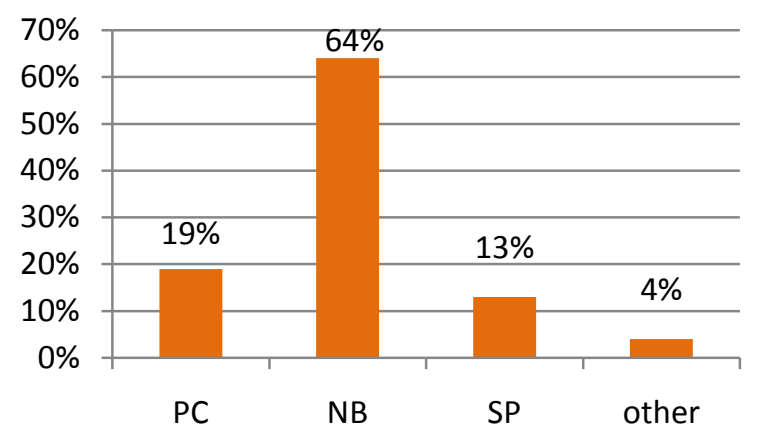

Fig. 1 Respondents using respective ICT elements [\%]

Even though further development of ICT may have a stimulating effect on an expansion of an IF, ICT elements may also offer new possibilities for a solution. Three basic strategies on how to approach IF can be distinguished. (49) They are [8,9]:
- organization strategy
- the user himself creates a set of rules for information processing and follows them 
- $\quad$ technical strategy

$\circ$ if the user receives data and information with a suitable structure, he can apply advanced functions of devices for an automatization of information processes and activities

- $\quad$ software strategy

$\circ$ the user applies specialized software in order to sustain data consistency required for further information processes.

The last two listed strategies are based on the application of ICT. The following figure (Fig. 2.) shows the percentage of users by the strategy they apply.

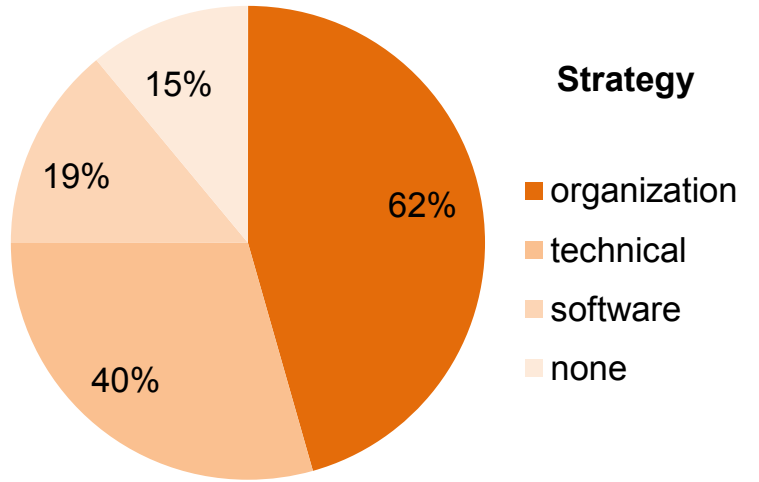

Fig. 2. Strategies applied by users [\%].

The previously mentioned conducted survey was also aimed at the proportions of the used strategies among the respondents. The survey revealed that many users do not consider IF to be an important issue, only $15 \%$ of users do not apply any strategy for coping with an IF.

Also, only $4 \%$ of the surveyed users use a form of a technical strategy for coping with an IF, probably due to requirements and limitations of this strategy. In a similar manner, a high percentage of users who apply the organization strategy may be based on its uncomplicated inclusion into everyday activities.

The collected data were subjected to a cluster analysis. The cluster analysis considered the following variables:

a) cloud storage

b) number of various data storages

c) level of duplication (how many backups of one file or project user creates)

d) how many various types of files user creates

e) how many various devices user applies

In the following graphs (Fig. 3. and Fig. 4.) we can see the exact values of these variables. Detected clusters are marked Cl.1-Cl.4.The letters a-e represent corresponding variables as listed before. For better orientation both graphs include variable c) level of duplication.

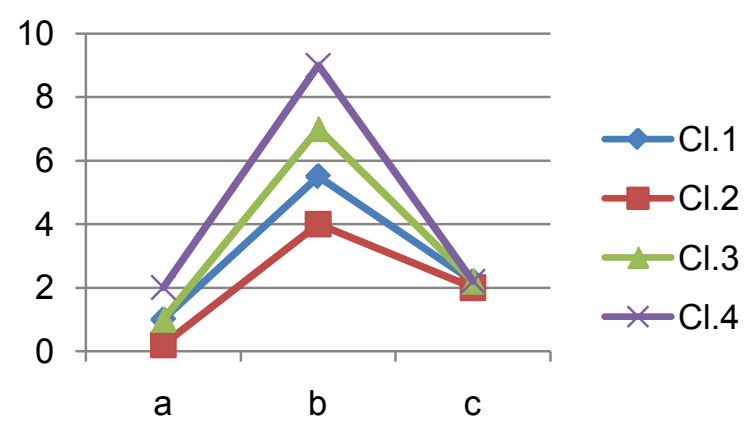

Fig. 3. The first part of the cluster analysis results.

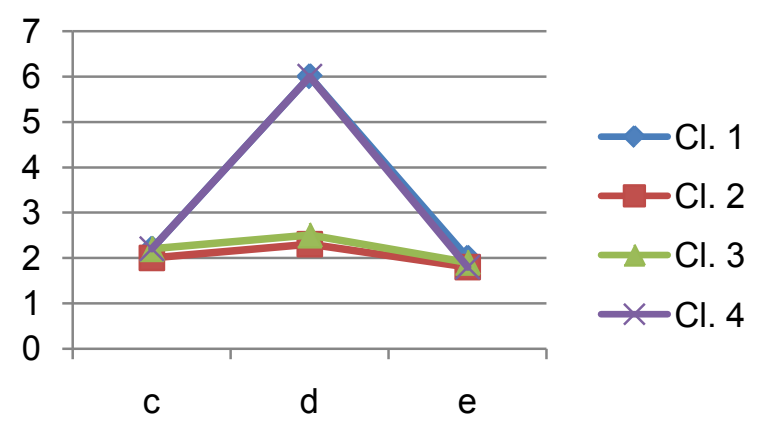

Fig. 4 The second part of the cluster analysis results.

From presented data, we can clearly see attributes, presented here as variables, that are the most similar between users and that are vastly different between groups of users. In an examined variable c) level of duplication we can see that the value of this variable is closely approaching a value of 2 , meaning that users in each individual group use approximately 2 backup copies of their files. This variable doesn't represent a character of said backup if it is a local storage, cloud or any other form. The question of storage variability is answered by variables a) cloud storage that represents how many various cloud storages user utilizes and variable b) number of various data storages that show how many individual data storing devices is used. As the most common reason for a number of used data storages asked users reported "personal opinion". It is quite peculiar, that most users agree on 2 backups of their data. This phenomenon could be concerned for future research.

The biggest difference between detected clusters of users was in values of variable $d$ ) how many various types of files the user creates. Where $2^{\text {nd }}$ and $3^{\text {rd }}$ clusters (further named as conservative and technologic type) reach values smaller than 2.5 , the other clusters are at the value of 6 . Users from these clusters $(\mathrm{Cl} .1$ and $\mathrm{Cl}$. 4, further presented as proactive and systematic type) mostly reported their hobbies and personal activity (mostly nonfull-time employment or private business activities). This irregularity may be caused by uneven distribution of the sample. The selected students were from the same education programme and therefore share some personal attributes. A larger sample with more variety between users would be required to further examine this finding. 
Based on the cluster analysis of the collected data, four basic types of users were established and sorted by their approach. They are [8]:

- Proactive type (Cl.1)

O Organization IF coping strategy is mainly used by these users. They use ICT moderately, are aware of IF and can flexibly react to such problems

while maintaining focus on their objectives.

- Conservative type (Cl.2)

$\circ \quad$ These users rarely apply ICT and they rely more on their own experiences with a bit of the organization strategy. To prevent loss of functionality due to a failure of any personal information environment element, they utilize various forms of archiving and they are cautious during information processes and activities.

- $\quad$ Technologic type (Cl.3)

- Software and organization IF coping strategies are most commonly used among these users. They are aware of certain risks connected to IF and rely on the used ICT elements when dealing with such problems.

- $\quad$ Systematic type (Cl.4)

○ Users of this type apply the organization strategy of dealing with IF with a notable ICT support which they use for the purposes of information centralization and synchronization between various data storages.

Percentages of respondents and their user types are shown in the following figure (Fig. 5). Different types of users are marked:

- $\quad \mathrm{P}$ - proactive type

- $\quad$ C - conservative type

- $\quad$ T - technologic type

- $\quad \mathrm{S}$ - systematic type

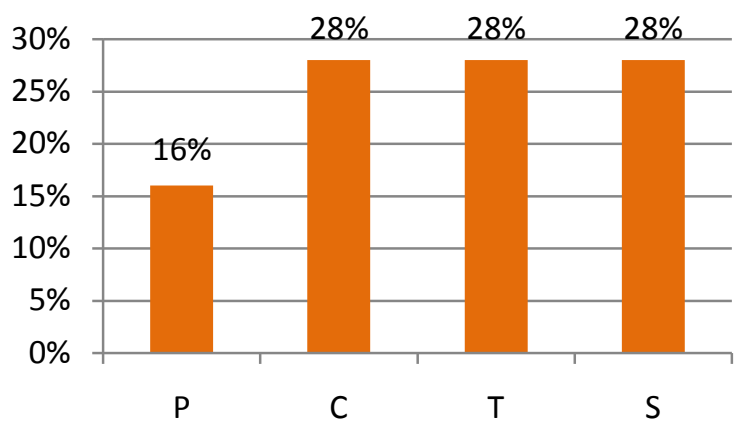

Fig. 5 Percentages of users by type [\%]
The conducted survey offers a possibility of creating user typology based on their approach to the IF phenomenon, thus opening new options for a personal information environment structure study.

\section{Discussion}

Integration of ICT into modern society and its organizations can be observed by means discussed in this article. The conducted study was focused on the problem of IF and PIE. These areas were explored by means of a survey. The main objective was to detect any structural groups amongst common users. By suitable analysis of the collected data it was possible to distinguish such groups in both areas of interest - IF and PIE. Revealed groups unite users by their strategy of dealing with an IF and their view of the discussed problem. Due to the characteristic of this field, an inclusion of ICT into a PIE was especially observed. The study revealed that even though certain elements of ICT are used increasingly, their advanced functions are still overlooked by a common user. However, we should bear in mind that this study was conducted on a highly specific sample of the population (university students of informatics). Larger groups with higher user diversity may yield different results.

The discussed study helps create suitable foundations for such future studies. By exploring these phenomena, an improvement in technical design can be achieved, thus supporting human-computer interaction and integration of ICT in general. Security is one of the most discussed fields considering PIE, where it could be used to reduce human error.

\section{References}

1. Official website of Czech statistical office, Information society in figures - 2010 (2010)

2. Official website of Czech statistical office, Information society in figures - 2011 (2011)

3. Official website of Czech statistical office, Information society in figures - 2013 (2013)

4. Official website of Czech statistical office, Information society in figures - 2015 (2015)

5. W. J. Martin, The global information society (1996)

6. J. Berce, S. Lanfranco, E-Governmence: Handbook of Research on E-Government readiness for information and service exchange (2010)

7. M. Hilbert, Digital Technology and Social Change (2015)

8. M. Čunderlová, Typologie informační fragmentace (2016)

9. W. P. Jones, Keeping found things found: the study and practice of personal information management (2008) 